\title{
Growth and electronic structure of nitrogen-doped graphene on $\mathrm{Ni}(111)$
}

\author{
R. J. Koch, ${ }^{1}$ M. Weser, ${ }^{2}$ W. Zhao, ${ }^{3}$ F. Viñes, ${ }^{4}$ K. Gotterbarm, ${ }^{3}$ S. M. Kozlov, ${ }^{4}$ O. Höfert, ${ }^{3}$ M. Ostler, ${ }^{1}$ C. Papp,${ }^{3,}$ \\ J. Gebhardt, ${ }^{4}$ H.-P. Steinrück, ${ }^{3}$ A. Görling, ${ }^{4}$ and Th. Seyller ${ }^{1, \dagger}$ \\ ${ }^{1}$ Lehrstuhl für Technische Physik, Universität Erlangen-Nürnberg, 91058 Erlangen, Germany \\ ${ }^{2}$ Fritz-Haber-Institut der Max-Planck-Gesellschaft, 14195 Berlin, Germany \\ ${ }^{3}$ Lehrstuhl für Physikalische Chemie II, Universität Erlangen-Nürnberg, 91058 Erlangen, Germany \\ ${ }^{4}$ Lehrstuhl für Theoretische Chemie, Universität Erlangen-Nürnberg, 91058 Erlangen, Germany
}

(Received 14 June 2012; published 1 August 2012)

\begin{abstract}
We report on experimental and theoretical investigations of nitrogen-doped graphene. The incorporation of nitrogen was achieved during chemical-vapor deposition on $\mathrm{Ni}(111)$ using pyridine as a precursor. The obtained graphene layers were investigated using photoelectron spectroscopy. By studying $\mathrm{C} 1 s$ and $\mathrm{N} 1 s$ core levels, we show that the nitrogen content is influenced by the growth temperature and determine the atomic arrangement of the nitrogen atoms. Valence-band photoelectron spectra show that the incorporation of nitrogen leads to a broadening of the photoemission lines and a shift of the $\pi$ band. Density functional calculations for two possible geometric arrangements, the substitution of carbon atoms by nitrogen and vacancies in the graphene sheet with pyridinic nitrogen at the edges, reveal that the two arrangements have opposite effects on the band structure. For the present experimental approach, vacancies with pyridinic nitrogen are dominant. In the latter case the vacancies generated by the nitrogen doping, not the nitrogen itself, have the main effect on the band structure. By intercalating gold between the doped graphene layer and the $\mathrm{Ni}(111)$ substrate electronic decoupling is achieved. After intercalation the doping remains.
\end{abstract}

DOI: 10.1103/PhysRevB.86.075401

PACS number(s): 73.22.Pr, 79.60.Dp, 71.15.Mb

\section{INTRODUCTION}

Graphene, a two-dimensional sheet of $s p^{2}$-bonded carbon atoms in a honeycomb arrangement, is one of the most exciting materials currently under investigation. Graphene has been recognized for its fascinating physical properties which arise from the linear dispersion relation of its massless, chiral charge carriers and for its high potential for applications. ${ }^{1,2}$ During the last years, much work has been devoted to the development of methods for large-scale synthesis of graphene following various approaches, such as sublimation growth on silicon carbide $^{3-7}$ or growth on metal substrates. ${ }^{8-13}$ In addition, it is also desirable to be able to modify the properties of graphene. Doping is a particularly important aspect for the purpose of adjusting the carrier concentration and type, which is a step forward towards the realization of graphene transistors ${ }^{14}$ and $p-n$ junctions. ${ }^{15}$ The doping of graphene has previously been achieved by charge transfer from adsorbed molecules ${ }^{16-18}$ or atoms, ${ }^{19-21}$ for example. In semiconductor technology, the term doping is reserved for a controlled contamination of the material by inserting impurities in the host lattice. The doping of graphene in this traditional sense by, e.g., the substitution of carbon by nitrogen or boron, may be an interesting tool for tailoring the electronic properties of graphene for electronic devices. Chemically induced mobility gaps were theoretically predicted upon the incorporation of boron impurities in graphene nanoribbons (GNRs), which could open a path to better on/off ratios in GNR-based transistors. ${ }^{22}$ In contrast to charge-transfer doping by adsorbates, substitutional doping is expected to be more stable against physical and chemical changes in the environment, thus making it very favorable for the use under ambient conditions. Furthermore, substitutional doping is also attractive for other reasons. For example, there is experimental evidence that nitrogen-doped graphene may be an interesting catalyst for fuel cells. ${ }^{23}$ Furthermore, impurities and impurity complexes may create the opportunity for the chemical functionalization of graphene sheets. On the other hand, defects and impurities constitute scattering centers which are expected to play an important role in the electronic transport in graphene. A detailed understanding of the effects of such scattering centers is of the utmost importance. . $21,24,25^{2}$ Nitrogen dopants constitute point defects, ${ }^{26,27}$ which facilitate a further investigation of the effects of defects on the ballistic transport in graphene.

Here, we investigate $\mathrm{N}$-doped graphene by a combination of photoelectron spectroscopy and theoretical calculations. We show that nitrogen-doped graphene can be grown on $\mathrm{Ni}(111)$ by chemical-vapor deposition (CVD) in an ultrahigh-vacuum environment using pyridine $\left(\mathrm{C}_{5} \mathrm{H}_{5} \mathrm{~N}\right)$ as a precursor. As the effect of the introduction of nitrogen atoms on the electronic properties of graphene might be masked by the well-known strong interaction of graphene with $\mathrm{Ni}(111)$, we have also investigated the layers after the intercalation of gold, which is known to decouple graphene from its substrate. ${ }^{28}$ Changes of the graphene band structure upon doping in the strongly coupled (without $\mathrm{Au}$ ) and decoupled (with $\mathrm{Au}$ ) states are interpreted with the help of ab initio density functional (DF) calculations. We show that the effect of doping remains present after Au intercalation.

\section{EXPERIMENTAL METHODS}

The experiments were carried out using synchrotron radiation at BESSY II, Berlin, Germany. For the angleresolved photoelectron spectroscopy (ARPES) measurements we used a toroidal energy analyzer. ${ }^{29}$ For x-ray photoelectron spectroscopy (XPS) measurements, a hemispherical energy analyzer EA125 was used. ${ }^{30}$ Both chambers were equipped with a dedicated preparation chamber in which the $\mathrm{Ni}(111)$ 


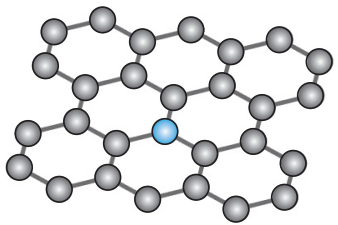

(a) substitutional (b) pyridinic

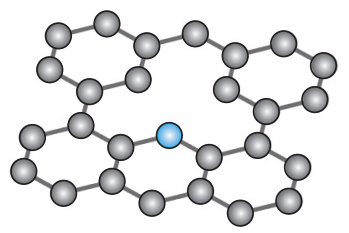

FIG. 1. (Color online) The two different structures for nitrogen (blue) incorporated in a graphene sheet considered in the present work: (a) substitutional sites and (b) pyridinic sites. The latter consist of a nitrogen atom accompanied by an adjacent vacancy.

crystal was cleaned before every experiment using standard procedures. ${ }^{31}$ The nitrogen-doped graphene was produced by exposing the sample to $1 \times 10^{-5}$ Torr pyridine at temperatures of 400,580 , or $800^{\circ} \mathrm{C}$ for 2 minutes, leading to nitrogen concentrations of $0.05,0.02$, or 0.00 monolayers (ML), respectively (determined by XPS). The intercalation of gold was achieved by evaporating $2 \mathrm{ML}$ of gold at room temperature and subsequently annealing the sample to $500^{\circ} \mathrm{C}$.

The DF calculations employed the VASP program package ${ }^{32}$ and projector-augmented-waves (PAWs) ${ }^{33}$ for the description of the core electrons. The Perdew-Burke-Erzenhof ${ }^{34}$ (PBE) exchange-correlation functional plus a dispersion correction to take into account van der Waals (vdW) interactions was used. ${ }^{35}$ This approach has been proven to adequately describe the interaction of graphene with metal surfaces. ${ }^{31,36,37}$ Different graphene $p(N \times N)$ unit cells $(N=2,4,6,8)$ have been used to model different nitrogen concentrations $(0.25,0.063,0.028$, and $0.016 \mathrm{ML})$. The graphene-Ni geometry was optimized in a six-layer slab in a $p(1 \times 1)$ cell with the bottom three layers fixed at the nickel bulk geometry. Calculations were carried out in the bridge-top geometry that was found to be the energy minimum in earlier work. ${ }^{31}$ The gold intercalation $[\mathrm{Au} / \mathrm{Ni}(111)]$ was modeled by introducing one layer of gold atoms in a $p(2 \times 2)$ cell of the $\mathrm{Ni} /$ graphene system. This cell contains a gold coverage of 0.75 ML. ${ }^{38}$ For further investigation, the geometries in the larger unit cells were kept fixed, and the four bottom nickel layers were removed. Optimized Monkhorst-Pack $k$-point grids of $21 \times 21 \times 1$ and $13 \times 13 \times 1$ have been used for the $p(2 \times 2)$ cells of graphene on $\mathrm{Au} / \mathrm{Ni}(111)$ and graphene on $\mathrm{Ni}(111)$, respectively. For these $k$-point grids and the ones chosen accordingly for the larger cells, the band structure was determined to be converged.

We have considered two possible geometric arrangements for nitrogen in graphene, which are shown in Fig. 1. Figure 1(a) shows the substitutional site $\left(\mathrm{N}_{s}\right)$, in which a $\mathrm{C}$ atom is replaced by a $\mathrm{N}$ atom, and Fig. 1(b) shows a pyridinic configuration $\left(\mathrm{N}_{\mathrm{py}}\right)$, i.e., a complex consisting of a $\mathrm{N}$ atom on a substitutional site accompanied by a vacancy. Other geometric arrangements may exhibit larger holes, i.e., more missing carbon atoms, and may contain more nitrogen atoms at the edges of a hole.

\section{RESULTS AND DISCUSSION}

Figure 2(a) shows the band structure of graphene on $\mathrm{Ni}(111)$ which was produced with the precursor pyridine at a substrate temperature of $T_{s}=800^{\circ} \mathrm{C}$. XPS analysis of the layer confirmed that it contains no nitrogen, and it is therefore no surprise that the data matches previous reports on graphene on $\mathrm{Ni}(111)$ synthesized from propene (see, e.g., Ref. 28 and references therein). Apparently, at that deposition temperature no nitrogen is built in. One may speculate that this is due to a temperature-induced decomposition of the pyridine precursor followed by the recombination and desorption of molecular nitrogen. The Ni $d$ bands are observed at binding energies between the Fermi level $E_{B}=E_{F}=0 \mathrm{eV}$ and $E_{B}=6 \mathrm{eV}$ (Refs. 39-41) in agreement with our calculation [see Fig. 2(e)]. The graphene $\pi$ band displays the well-known pronounced dispersion and the band-gap opening at the $K$ point due to the electronic coupling of the carbon $\pi$ states to the $\mathrm{Ni} d$ bands of appropriate symmetry. ${ }^{36,37,42,43}$ The $\sigma$ bands are well resolved in the whole Brillouin zone, showing the expected dispersion in accordance with the DF calculations [see the orange bands in Fig. 2(e)]. Corresponding measurements of graphene grown by pyridine CVD at a substrate temperature of $T_{s}=580^{\circ} \mathrm{C}$ are shown in Fig. 2(b). XPS measurements (see below) indicate a nitrogen content of $0.02 \mathrm{ML}$, i.e., 1\%. Qualitatively, the data are very similar to those shown in Fig. 2(a) for undoped graphene. Closer inspection of the data reveals small but significant and systematic differences, which will be discussed below.

Before we return to the nitrogen-induced variations in the valence band, we discuss the results of $\mathrm{Au}$ intercalation. Figure 3 depicts $\mathrm{C} 1 s, \mathrm{~N} 1 s$, and Ni $2 p$ core-level spectra of graphene grown by pyridine CVD at $T_{s}=580{ }^{\circ} \mathrm{C}$ before and after the intercalation of gold. The $\mathrm{C} 1 \mathrm{~s}$ spectrum of graphene/Ni(111) reveals a geometric arrangement of graphene with the carbon atoms predominantly adopting equivalent bridge-top sites, as discussed in a recent combined XPS and DF study. ${ }^{31}$ After gold intercalation, the C $1 s$ core level shifts significantly to lower binding energies by $0.7 \mathrm{eV}$ to a position comparable to freestanding graphene on other substrates. ${ }^{44,45}$ Comparing the $\mathrm{C} 1 s$ core level binding energies of graphite $\left(E_{B}=284.45 \mathrm{eV}\right)$ to that of our quasifreestanding graphene $\left(E_{B}=284.30 \mathrm{eV}\right)$, we find a shift of $150 \mathrm{meV}$. The $\mathrm{N} 1 s$ core level consists of two distinct contributions: $\mathrm{N}_{s}$ at a binding energy of $400.6 \mathrm{eV}$ and $\mathrm{N}_{\text {py }}$ at $399.0 \mathrm{eV}$, which are attributed to $\mathrm{N}$ atoms in substitutional and pyridinic configurations, respectively (compare with Fig. 1). While a significant damping due to the gold layer is observed for the $\mathrm{Ni}$ $2 p$ signal [see Fig. 3(c)], the $\mathrm{C} 1 s$ and $\mathrm{N} 1 s$ in Figs. 3(a) and 3(b) show no significant loss in intensity in agreement with Ref. 46. This is independent proof of the intercalation of Au underneath graphene. It, furthermore, demonstrates unambiguously that the nitrogen is incorporated in the graphene layer and not bound to $\mathrm{Ni}$ and that the intercalation procedure does not lead to a significant desorption of the nitrogen. The small changes in the ratio of the two nitrogen species are due to the thermal treatment during intercalation, leading to a transformation of $\mathrm{N}_{\text {py }}$ to $\mathrm{N}_{s} \cdot{ }^{46}$ Also, the $\mathrm{N} 1 s$ peak associated to pyridinic nitrogen shifts after gold intercalation while the $\mathrm{N} 1 s$ peak associated to substitutional nitrogen does not shift. This can be explained by the stronger substrate interaction of the pyridinic nitrogen, which is prevented by gold intercalation.

Now we return to the discussion of the valence bands. ARPES data of nitrogen-free and nitrogen-doped graphene 
(a)

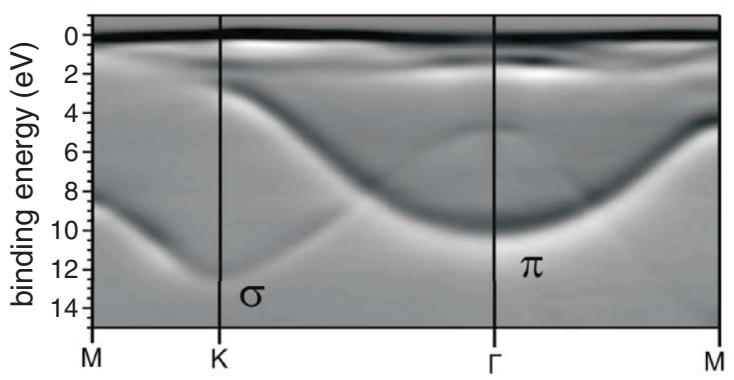

(c)

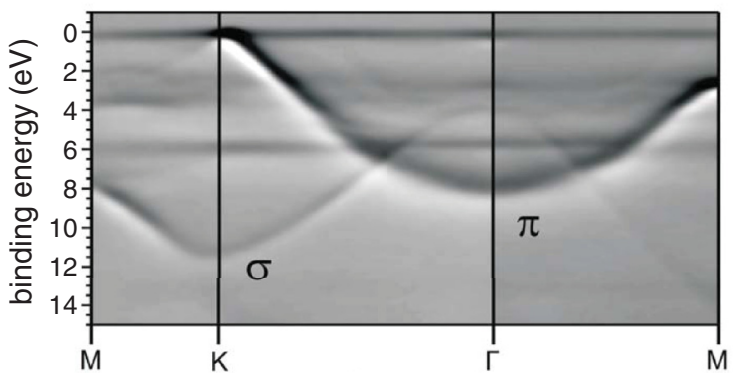

(e)

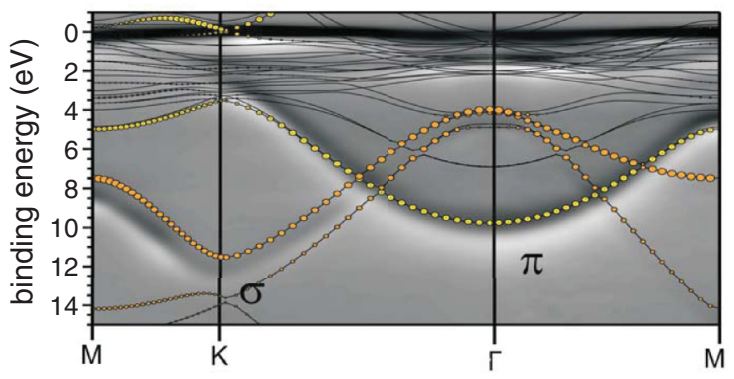

(b)

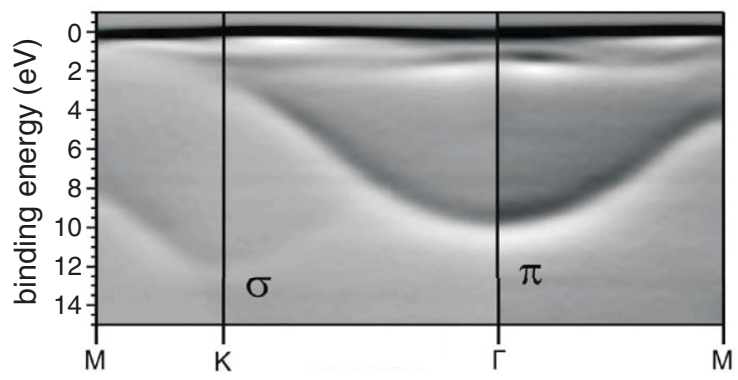

(d)

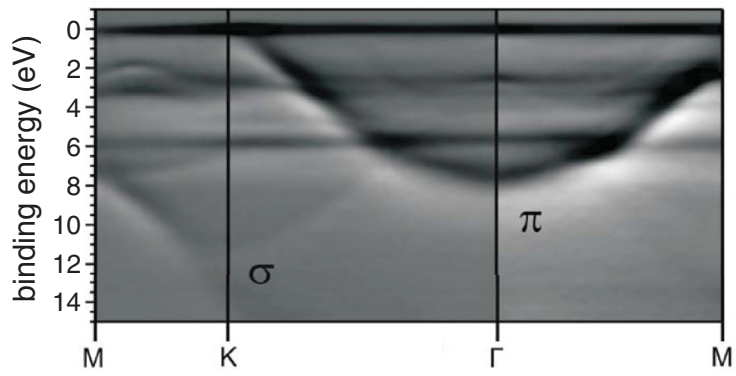

(f)

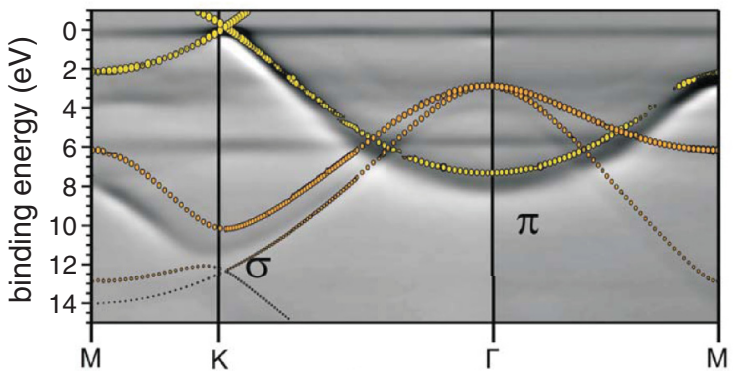

FIG. 2. (Color online) Differentiated photoelectron intensity maps vs. binding energy and parallel momentum of graphene grown by pyridine CVD at (a) $800^{\circ} \mathrm{C}$ and (b) $580^{\circ} \mathrm{C}$, resulting in nitrogen contents of $0.00 \mathrm{ML}$ and $0.02 \mathrm{ML}$, respectively. (c) and (d) display data taken from the samples shown in (a) and (b) after gold intercalation. The photon energy was $h v=40 \mathrm{eV}$. (e) and (f) band structures from DF calculations (black lines) superimposed on the photoemission data of (a) and (c), nitrogen-free graphene on $\mathrm{Ni}(111)$ and $\mathrm{Au} / \mathrm{Ni}(111)$, respectively. Note that in (f), for a better comparison with the experimental results, the band structure calculated in a $p(2 \times 2)$ supercell is unfolded to be comparable to band structures referring to the $p(1 \times 1)$ unit cell. Yellow and orange dots denote the carbon $p_{z}$ and $p_{x, y}$ contributions (corresponding to the dot size) for the given band, respectively.

on $\mathrm{Ni}(111)$ after intercalation with $\mathrm{Au}$ are presented in Figs. 2(c) and 2(d), respectively. In agreement with previous investigations, ${ }^{28}$ the $\mathrm{Ni} d$ bands are strongly suppressed by the Au layer which in turn manifests itself by the almost dispersionless Au $d$ bands visible between the 4-6 eV binding energy. After the intercalation of Au, the graphene $\pi$ band shifts by approximately $2 \mathrm{eV}$ towards the Fermi level while the $\sigma$ band shows a shift of only $1.3 \mathrm{eV}$. Similar shifts of 2.1 and $1.1 \mathrm{eV}$ for the $\pi$ and $\sigma$ bands, respectively, are found in the calculated band structure upon Au intercalation. As an example, we show the calculated band structures for the nitrogen-free case superimposed on the ARPES measurements in Figs. 2(e) and 2(f). The calculated band structures agree well but not perfectly with the measured ones. The largest discrepancy of about $2 \mathrm{eV}$ occurs for the $\sigma$ band at the $K$ point and is typical in magnitude for DF band structures. Higher accuracies could perhaps be achieved by calculations with computationally much more demanding many-body perturbation approaches like the GW method. ${ }^{47}$
The $\pi$ band shifts stronger than the $\sigma$ band because of its hybridization with the $\mathrm{Ni} d$ bands, which is turned off by the Au intercalation. This behavior is also reflected in the carbon contributions of the calculated bands before and after intercalation, which are made visible via the sizes of the dots of the calculated $\sigma$ and $\pi$ bands in Figs. 2(e) and 2(f). Before intercalation, the $\sigma$ band has contributions mainly coming from the graphene carbon atoms, while the $\pi$ band has smaller contributions from the carbon atoms and, therefore, more contributions from the states of the Ni substrate. ${ }^{40,41}$ After gold intercalation, when the $\pi$ band has recovered the linear dispersion around the $K$ point, the contribution of the carbon atoms increases and that of the Ni $d$ states decreases. This confirms the change towards quasifreestanding graphene. In nitrogen-doped graphene, the energy dispersion of the $\pi$ band between the $\Gamma$ and the $M$ points is smaller by approximately $0.25 \mathrm{eV}$ for a nitrogen coverage of $0.02 \mathrm{ML}$. This change is about $5 \%$ of the total energy dispersion of $5.6 \mathrm{eV}$. A similar observation is made for the $\sigma$ band between the $\Gamma$ and $K$ points, 

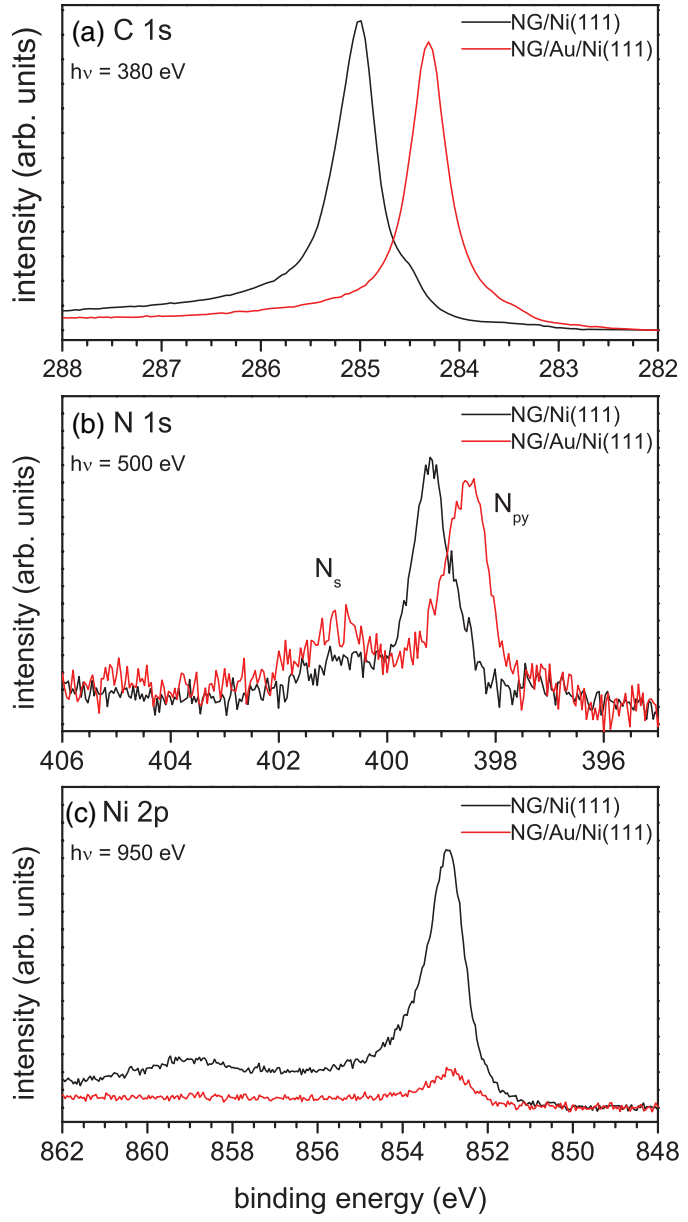

FIG. 3. (Color online) High-resolution x-ray photoelectron spectra of the intercalation of nitrogen-doped graphene on $\mathrm{Ni}(111)$. Shown are the (a) $\mathrm{C} 1 s$, (b) $\mathrm{N} 1 s$, and (c) $\mathrm{Ni} 2 p$ regions.

which changes in width by around $0.4 \mathrm{eV}$. We think this change of the energy dispersion originates from a localization of the electronic states at the defects and nitrogen atoms.

Figure 4 depicts energy-dispersion curves (EDCs) at the $\Gamma$ point for the different preparations. The upper three spectra [Figs. 4(a)-4(c)] represent graphene with different nitrogen concentrations directly after growth, and the lower three spectra [Figs. 4(d)-4(f)] show the same samples after Au intercalation. Before Au intercalation, the graphene $\pi$ band at the $\Gamma$ point significantly shifts to smaller binding energies $(0.3 \mathrm{eV})$ when incorporating $0.02 \mathrm{ML}$ of nitrogen. The shift is even more pronounced $(0.6 \mathrm{eV})$ for a nitrogen concentration of $0.05 \mathrm{ML}$. After Au intercalation, the binding energy of the $\pi$ band decreases due to the transition to a more quasifreestanding nature as discussed above. In addition, there is still a dependence of the position of the bottom of the $\pi$ band on the nitrogen concentration. However, the changes in binding energy are smaller $(0.1 \mathrm{eV}$ and $0.3 \mathrm{eV}$ for $\mathrm{N}$ concentrations of $0.02 \mathrm{ML}$ and $0.05 \mathrm{ML}$, respectively). Please note that preparing graphene with propylene in the discussed temperature range does not lead to a shift of the $\pi$ band at the $\Gamma$ point (data not shown).

Figure 5 shows the calculated binding energy of the bottom of the $\pi$ band for different doping levels in the two geometrical

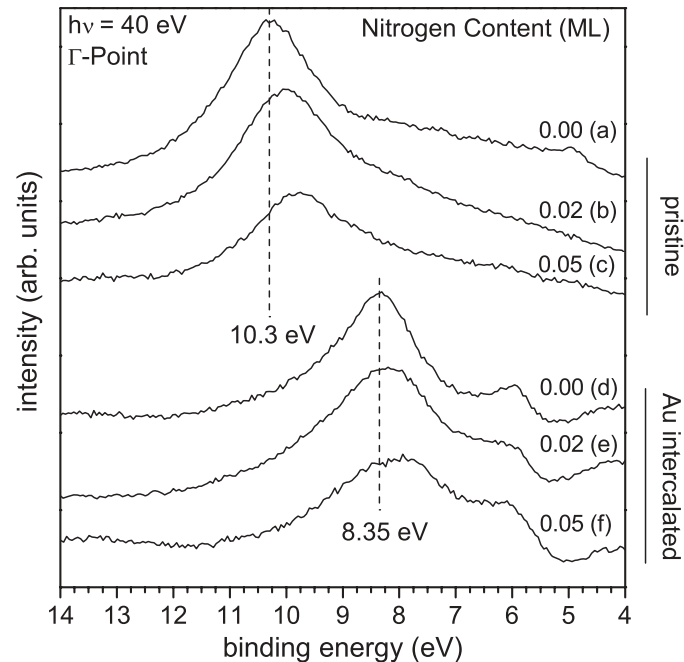

FIG. 4. Energy distribution curves (EDCs) of different ARPES spectra at the $\Gamma$ point. (a)-(c) Spectra from preparations at different temperatures. (d)-(f) Gold-intercalated graphene layers.

arrangements of nitrogen in graphene on $\mathrm{Ni}(111)$ as well as on $\mathrm{Au} / \mathrm{Ni}(111)$. For the latter case, the position of the Dirac point is shown as well. The calculations show that the position of the bottom of the $\pi$ band strongly depends on the nitrogen concentration and on the geometry. For the pyridinic configuration, the $\pi$ band is shifted to lower binding energies in agreement with the experimental observations, meaning that the nitrogen in our $\mathrm{N}$-doped graphene exists predominantly in the pyridinic form. This conclusion is

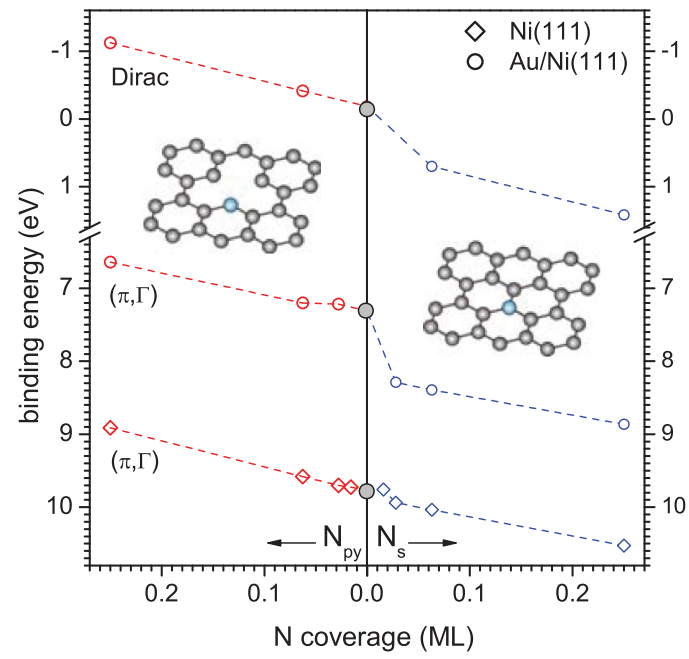

FIG. 5. (Color online) Binding energy of the bottom of the $\pi$ band of $\mathrm{N}$-doped graphene on $\mathrm{Ni}(111)$ and $\mathrm{Au} / \mathrm{Ni}(111)$ as a function of the concentration of pyridinic nitrogen $\left(\mathrm{N}_{\mathrm{py}}\right.$, left side) and substitutional nitrogen $\left(\mathrm{N}_{s}\right.$, right side). The position of the Dirac point is included for the $\mathrm{Au} / \mathrm{Ni}(111)$ case as well. Note that the Dirac cone is opened in our calculations of $\mathrm{N}$-doped graphene independently of the substrate or specific doping geometry due to the reduction of symmetry upon doping. Therefore, in these cases $E_{D}$ is set equal to the midpoint of the arising band gap at $K$. 
valid for both graphene on $\mathrm{Ni}(111)$ and on $\mathrm{Au} / \mathrm{Ni}(111)$ and is supported by the $\mathrm{N} 1 s$ core-level spectra. In the case of substitutional nitrogen, the calculations show that the bottom of the $\pi$ band is shifted towards higher binding energies, again, for both substrates. Note that this behavior is in qualitative agreement with calculations performed for freestanding graphene. ${ }^{48,49} \mathrm{~A}$ similar finding was also made for the Dirac point of graphene on $\mathrm{Au} / \mathrm{Ni}(111)$. An extrapolation of the $\pi$ band to the $K$ point shows that the Dirac point is above the Fermi level in agreement with the calculations. We also note that for the quasifreestanding case, the effect of doping on the position of the $\pi$ band is stronger for the substitutional configuration than for the pyridinic one. As mentioned above, the intercalation leads to a certain degree of conversion of $\mathrm{N}_{\text {py }}$ to $\mathrm{N}_{s}$. Therefore, the observed overall shift of the $\pi$ band is reduced compared to the nonintercalated samples.

For the pyridinic configuration, which is composed of a combination of a nitrogen atom on a substitutional site and a vacancy next to it, we may ask to what extent the replacement of carbon by nitrogen and to what extent the vacancy is responsible for the effect on the electronic structure. Hence, we have also carried out calculations for vacancies alone, which showed that the latter are responsible for typically (80-90)\% of the observed shifts of the $\pi$ band.

In a recent study, Usachov et al $^{46}$ grew N-doped graphene on $\mathrm{Ni}(111)$ using $s$-triazine $(\mathrm{HCN})_{3}$. In their case, the nitrogen atoms were predominantly incorporated in the substitutional configuration. Consequently, they observed a shift of the $\pi$ band towards higher binding energies.

The broadening of the peaks in the ARPES spectra in Figs. 2 and 4 in the case of nitrogen-doped graphene is attributed to a statistical distribution of nitrogen atoms in the graphene layer, which can be considered as defects of the periodic lattice. ${ }^{26}$ They induce a multitude of new states, occurring around the original $\pi$ and $\sigma$ bands of graphene, leading to a broadening of up to $0.6 \mathrm{eV}$ [see Fig. 4(f)].

The energetic stability of the two doping geometries, substitutional and pyridinic nitrogen, was investigated by DF calculations for doping concentrations of $0.056 \mathrm{ML}$ and 0.031 ML. Estimates by the DF calculations show that thermodynamically both nitrogen substitutions are markedly endothermic by at least $2.75 \mathrm{eV}$. The energies were calculated by replacing a graphene carbon atom by a nitrogen atom in pristine graphene or in graphene with a carbon vacancy, respectively, and by subsequently taking the difference between the energy of graphene (or graphene with a vacancy) plus an isolated nitrogen atom minus the energy of nitrogen-doped graphene (substitutional or pyridinic) plus an isolated carbon atom. In the presence of a carbon vacancy, a doping arrangement with pyridinic nitrogen, i.e., a nitrogen adjacent to the vacancy, is favored over substitutional nitrogen, i.e., a nitrogen not neighboring the vacancy, by $0.82-1.07 \mathrm{eV}$, depending on the size of the unit cell. However, the formation of pyridinic nitrogen requires the formation of a defect, which is less stable than an intact graphene layer, making substitutional nitrogen the thermodynamic more stable geometry. This might allow for control of the geometrical arrangement in nitrogen-doped graphene by the growth conditions, i.e., the formation of carbon vacancies during synthesis or post annealing. ${ }^{46}$ The creation of carbon defects and their saturation with nitrogen might be one desired route to tune the electronic properties of graphene on strongly coupling substrates.

Additionally, calculations on the $p(3 \times 3)$ unit cell show that carbon present in the subsurface region-in octahedral subsurface sites - of the Ni(111) crystal may be thermodynamically driven out of the substrate, healing the carbon-vacancy defect in the pyridinic case. For the considered supercell, graphene with a substitutional nitrogen is calculated to be $2.58 \mathrm{eV}$ more stable than an arrangement of graphene with a pyridinic nitrogen, an adjacent defect in the graphene network (see Fig. 1), and an extra carbon atom located in an octahedral subsurface site in the first subsurface layer of the Ni(111) surface. The healing of carbon vacancies by subsurface carbon by annealing might be a way to influence the geometry of nitrogen incorporated in graphene. ${ }^{46,50}$

\section{CONCLUSION}

In conclusion, we have shown that it is possible to grow nitrogen-doped graphene on a Ni(111) surface with different dopant concentrations and structural configurations. This is achieved by means of a CVD process using pyridine as a precursor and carrying out the synthesis at different substrate temperatures. The incorporation of nitrogen into the graphene network is shown by means of ARPES and XPS. Two different types of geometric arrangements, i.e., substitutional nitrogen and pyridinic nitrogen, are found. The observed effects, in particular a shift of the graphene $\pi$ band, are in line with $a b$ initio DF calculations. Different geometric situations, substitutional nitrogen versus pyridinic nitrogen, show an opposite effect on the energetic position of the Dirac cone. The calculations also show that substitutional nitrogen is the energetically most favorable situation although pyridinic nitrogen can be kinetically stable. Generally, the effect of nitrogen incorporation will depend on the ratio of substitutional and pyridinic nitrogen. Gold intercalation was used to decouple graphene and nitrogen-doped graphene from the $\mathrm{Ni}(111)$ substrate to determine the energy position of the Dirac point and to verify the incorporation of nitrogen in the graphene layer.

\section{ACKNOWLEDGMENTS}

The authors gratefully acknowledge the funding of the BMBF through Grant No. 05ES3XBA15 and the German Research Council (DFG), which supports the Collaborative Research Center 953 and which supports, within the framework of its Excellence Initiative, the Cluster of Excellence "Engineering of Advanced Materials" (www.eam.unierlangen.de) at the University of Erlangen-Nuremberg. We thank Karsten Horn for a critical reading of the manuscript. F.V. thanks the Alexander von Humboldt Foundation for financing his postdoctoral grant. We thank the BESSY staff, especially B. Mahler and W. Zada at UE56-2, for their support during beam time. W.Z. thanks the China Scholarship Council for financing his Ph.D. grant. 
*christian.papp@chemie.uni-erlangen.de

† thomas.seyller@physik.uni-erlangen.de

${ }^{1}$ A. K. Geim, Science 324, 1530 (2009).

${ }^{2}$ A. K. Geim and K. S. Novoselov, Nat. Mater. 6, 183 (2007).

${ }^{3}$ C. Berger, Z. M. Song, T. B. Li, X. B. Li, A. Y. Ogbazghi, R. Feng, Z. T. Dai, A. N. Marchenkov, E. H. Conrad, P. N. First, and W. A. de Heer, J. Phys. Chem. B 108, 19912 (2004).

${ }^{4}$ C. Berger, Z. Song, X. Li, X. Wu, N. Brown, D. Maud, C. Naud, and W. A. de Heer, Phys. Status Solidi A 204, 1521 (2007).

${ }^{5}$ K. V. Emtsev, A. Bostwick, K. Horn, J. Jobst, G. L. Kellogg, L. Ley, J. L. McChesney, T. Ohta, S. A. Reshanov, J. Roehrl, E. Rotenberg, A. K. Schmid, D. Waldmann, H. B. Weber, and T. Seyller, Nat. Mater. 8, 203 (2009).

${ }^{6}$ C. Virojanadara, M. Syväjarvi, R. Yakimova, L. I. Johansson, A. A. Zakharov, and T. Balasubramanian, Phys. Rev. B 78, 245403 (2008).

${ }^{7}$ P. N. First, W. A. de Heer, T. Seyller, C. Berger, J. A. Stroscio, and J.-S. Moon, MRS Bull. 35, 296 (2010).

${ }^{8}$ Q. Yu, J. Lian, S. Siriponglert, H. Li, Y. P. Chen, and S.-S. Pei, Appl. Phys. Lett. 93, 113103 (2008).

${ }^{9}$ X. Li, W. Cai, J. An, S. Kim, J. Nah, D. Yang, R. Piner, A. Velamakanni, I. Jung, E. Tutuc, S. K. Banerjee, L. Colombo, and R. S. Ruoff, Science 324, 1312 (2009).

${ }^{10}$ X. Li, Y. Zhu, W. Cai, M. Borysiak, B. Han, D. Chen, R. D. Piner, L. Colombo, and R. S. Ruoff, Nano Lett. 9, 4359 (2009).

${ }^{11}$ J. Wintterlin and M.-L. Bocquet, Surf. Sci. 603, 1841 (2009).

${ }^{12}$ K. S. Kim, Y. Zhao, H. Jang, S. Y. Lee, J. M. Kim, K. S. Kim, J.-H. Ahn, P. Kim, J.-Y. Choi, and B. H. Hong, Nature (London) 457, 706 (2009).

${ }^{13}$ S. Bae, H. Kim, Y. Lee, X. Xu, J.-S. Park, Y. Zheng, J. Balakrishnan, T. Lei, H. R. Kim, Y. I. Song, Y.-J. Kim, K. S. Kim, B. Ozyilmaz, J.-H. Ahn, B. H. Hong, and S. Iijima, Nat. Nanotechnol. 5, 574 (2010)

${ }^{14}$ X. Wang, X. Li, L. Zhang, Y. Yoon, P. K. Weber, H. Wang, J. Guo, and H. Dai, Science 324, 768 (2009).

${ }^{15}$ V. V. Cheianov, V. Fal'ko, and B. L. Altshuler, Science 315, 1252 (2007).

${ }^{16}$ W. Chen, S. Chen, D. C. Qi, X. Y. Gao, and A. T. S. Wee, J. Am. Chem. Soc. 129, 10418 (2007).

${ }^{17}$ C. Coletti, C. Riedl, D. S. Lee, B. Krauss, L. Patthey, K. von Klitzing, J. H. Smet, and U. Starke, Phys. Rev. B 81, 235401 (2010).

${ }^{18}$ S. Lara-Avila, K. Moth-Poulsen, R. Yakimova, T. Björnholm, V. Fal'ko, A. Tzalenchuk, and S. Kubatkin, Adv. Mat. (Weinheim, Ger.) 23, 878 (2011).

${ }^{19}$ T. Ohta, A. Bostwick, T. Seyller, K. Horn, and E. Rotenberg, Science 313, 951 (2006).

${ }^{20}$ A. Bostwick, T. Ohta, T. Seyller, K. Horn, and E. Rotenberg, Nat. Phys. 3, 36 (2007).

${ }^{21}$ J.-H. Chen, C. Jang, S. Adam, M. S. Fuhrer, E. D. Williams, and M. Ishgami, Nat. Phys. 4, 377 (2008).

${ }^{22}$ B. Biel, F. Triozon, X. Blase, and S. Roche, Nano Lett. 9, 2725 (2009).

${ }^{23}$ L. Qu, Y. Liu, J.-B. Baek, and L. Dai, ACS Nano 4, 1321 (2010).

${ }^{24}$ G. M. Rutter, J. N. Crain, N. P. Guisinger, T. Li, P. N. First, and J. A. Stroscio, Science 317, 219 (2007).
${ }^{25}$ A. Bostwick, J. L. McChesney, K. V. Emtsev, T. Seyller, K. Horn, S. D. Kevan, and E. Rotenberg, Phys. Rev. Lett. 103, 056404 (2009).

${ }^{26}$ L. Zhao, R. He, K. T. Rim, T. Schiros, K. S. Kim, H. Zhou, C. Gutiérrez, S. P. Chockalingam, C. J. Arguello, L. Pálová, D. Nordlund, M. S. Hybertsen, D. R. Reichman, T. F. Heinz, P. Kim, A. Pinczuk, G. W. Flynn, and A. N. Pasupathy, Science 333, 999 (2011).

${ }^{27}$ D. Geng, S. Yang, Y. Zhang, J. Yang, J. Liu, R. Li, T.-K. Sham, X. Sun, S. Ye, and S. Knights, Appl. Surf. Sci. 257, 9193 (2011).

${ }^{28}$ A. Varykhalov, J. Sánchez-Barriga, A. M. Shikin, C. Biswas, E. Vescovo, A. Rybkin, D. Marchenko, and O. Rader, Phys. Rev. Lett. 101, 157601 (2008).

${ }^{29}$ L. Broekman, A. Tadich, E. Huwald, J. Riley, R. Leckey, T. Seyller, K. Emtsev, and L. Ley, J. Electron Spectrosc. Relat. Phenom. 144147, 1001 (2005).

${ }^{30}$ R. Denecke, M. Kinne, C. Whelan, and H.-P. Steinrück, Surf. Rev. Lett. 9, 797 (2002).

${ }^{31}$ W. Zhao, S. M. Kozlov, O. Höfert, K. Gotterbarm, M. P. A. Lorenz, F. Viñes, C. Papp, A. Görling, and H.-P. Steinrück, J. Phys. Chem. Lett. 2, 759 (2011).

${ }^{32}$ G. Kresse and J. Furthmüller, Phys. Rev. B 54, 11169 (1996).

${ }^{33}$ P. E. Blöchl, Phys. Rev. B 50, 17953 (1994).

${ }^{34}$ J. P. Perdew, K. Burke, and M. Ernzerhof, Phys. Rev. Lett. 77, 3865 (1996).

${ }^{35}$ F. Ortmann, F. Bechstedt, and W. G. Schmidt, Phys. Rev. B 73, 205101 (2006)

${ }^{36}$ S. M. Kozlov, F. Viñes, and A. Görling, J. Phys. Chem. C 116, 7360 (2012).

${ }^{37}$ F. Mittendorfer, A. Garhofer, J. Redinger, J. Klimeš, J. Harl, and G. Kresse, Phys. Rev. B 84, 201401 (2011).

${ }^{38}$ M. H. Kang, S. C. Jung, and J. W. Park, Phys. Rev. B 82, 085409 (2010).

${ }^{39}$ A. Roldan, F. Viñes, F. Illas, J. M. Ricart, and K. M. Neyman, Theor. Chem. Acc. 120, 565 (2008).

${ }^{40}$ Y. S. Dedkov and M. Fonin, New J. Phys. 12, 125004 (2010).

${ }^{41}$ V. M. Karpan, P. A. Khomyakov, A. A. Starikov, G. Giovannetti, M. Zwierzycki, M. Talanana, G. Brocks, J. van den Brink, and P. J. Kelly, Phys. Rev. B 78, 195419 (2008).

${ }^{42}$ G. Bertoni, L. Calmels, A. Altibelli, and V. Serin, Phys. Rev. B 71, 075402 (2005).

${ }^{43}$ A. M. Shikin, G. V. Prudnikova, V. K. Adamchuk, F. Moresco, and K.-H. Rieder, Phys. Rev. B 62, 13202 (2000).

${ }^{44}$ K. V. Emtsev, F. Speck, T. Seyller, L. Ley, and J. D. Riley, Phys. Rev. B 77, 155303 (2008).

${ }^{45}$ F. Speck, J. Jobst, F. Fromm, M. Ostler, D. Waldmann, M. Hundhausen, H. B. Weber, and T. Seyller, Appl. Phys. Lett. 99, 122106 (2011).

${ }^{46}$ D. Usachov, O. Vilkov, A. Grüneis, D. Haberer, A. Fedorov, V. K. Adamchuk, A. B. Preobrajenski, P. Dudin, A. Barinov, M. Oehzelt, C. Laubschat, and D. V. Vyalikh, Nano Lett. 11, 5401 (2011).

${ }^{47}$ L. Hedin, Phys. Rev. 139, A796 (1965).

${ }^{48}$ B. Zheng, P. Hermet, and L. Henrard, ACS Nano 4, 4165 (2010).

${ }^{49}$ A. Lherbier, X. Blase, Y.-M. Niquet, F. Triozon, and S. Roche, Phys. Rev. Lett. 101, 036808 (2008).

${ }^{50}$ S. Karoui, H. Amara, C. Bichara, and F. Ducastelle, ACS Nano 4, 6114 (2010). 